\title{
CHK1 inhibition sensitizes pancreatic cancer cells to gemcitabine via promoting CDK-dependent DNA damage and ribonucleotide reductase downregulation
}

\author{
MIN LIANG, TIANGANG ZHAO, LINFENG MA and YINGJIE GUO
}

\author{
School of Life Sciences, Jilin University, Changchun, Jilin 130021, P.R. China
}

Received August 11, 2017; Accepted December 11, 2017

DOI: $10.3892 /$ or.2017.6168

\begin{abstract}
Inhibition of checkpoint kinase 1 (CHK1) is a promising therapeutic strategy to increase the effectiveness of DNA-damaging drugs in pancreatic cancer. However, owing to the multiple roles of CHK1 in the DNA damage response (DDR) pathway, the molecular mechanism of chemosensitization by CHK1 inhibitors is not definitive. In the present study, we explored the antitumor mechanism of LY2603618, a specific CHK1 inhibitor, alone or in combination with gemcitabine in 5 pancreatic cancer cell lines. LY2603618 treatment of the pancreatic cancer cell lines resulted in growth inhibition, with $\mathrm{IC}_{50}$ values ranging from 0.89 to $2.75 \mu \mathrm{M}$, but limited cell death. Importantly, treatment of pancreatic cancer cell lines with LY2603618 reduced the levels of pCDC25C, pCDK1, and pCDK2, accompanied by DNA damage and RRM1/2 downregulation. Furthermore, LY2603618 synergized with gemcitabine treatment to induce growth inhibition and apoptosis in pancreatic cancer cells. Mechanistic investigations showed that gemcitabine sensitization by CHK1 inhibition was associated with CDK-dependent RRM1/2 downregulation and DNA damage enhancement. These findings provide a basis for further development of combining CHK1 inhibitors and gemcitabine to treat pancreatic cancer.
\end{abstract}

\section{Introduction}

Pancreatic cancer is a highly malignant disease with a 5 -year survival rate of less than $4 \%$ (1). Gemcitabine (2'-deoxy-2',2'-difluorocytidine monohydrochloride) is the standard first-line drug used to treat patients with advanced pancreatic cancer (2). Its active metabolites, diphosphorylated and triphosphorylated nucleosides (dFdCDP and dFdCTP), inhibit both DNA polymerase and ribonucleotide reductase (RR), leading to impaired DNA synthesis and repair, and then cause DNA damage and apoptosis (3). However, its efficacy

Correspondence to: Professor Yingjie Guo, School of Life Sciences, Jilin University, 2699 Qianjin street, Changchun, Jilin 130021, P.R. China

E-mail: guoyingjie@jlu.edu.cn

Key words: CHK1 inhibition, gemcitabine, CDK, ribonucleotide reductase, DNA damage remains low with a median survival rate of 5.7 months and a 1-year survival rate of $18 \%(4,5)$. This has been attributed, in part, to the presence of a highly effective DNA damage response in pancreatic cancer.

Checkpoint kinase 1 (CHK1) acts as a master regulator of DNA damage signaling to regulate cell cycle progression, DNA repair, and DNA replication (6). CHK1 is activated by diverse stimuli including DNA-damaging agents via both ATM and Rad3-related (ATR) and ataxia telangiectasia-mutated (ATM). Activated CHK1 destablizes CDC25s (e.g., CDC25C) to prevent the activation of CDKs and cause cell cycle arrest (7). Inhibition of CHK1 abrogates DNA damage-induced cell cycle arrest allowing cells to enter mitosis despite the presence of DNA damage, which can lead to cell death, especially in p53-defective cancer cells. p53 gene is inactivated in 50 to $75 \%$ of pancreatic cancers (8). Thus inhibition of $\mathrm{CHK} 1$ is a promising cancer therapeutic strategy for increasing the chemosensitization in pancreatic cancer.

Numerous inhibitors of Chk1 are in pre-clinical and clinical development with the focus predominantly on their ability to potentiate the cytotoxicity of chemotherapy drugs. However, owing to the multiple roles of CHK1 in the DNA damage response (DDR) pathway, molecular mechanism of chemosensitization by CHK1 inhibitors is not definitive. Both the abrogation of $\mathrm{S}$ or $\mathrm{G} 2 / \mathrm{M}$ checkpoint and inhibition of homologous recombination repair (HRR) have been reported to contribute to chemosensitization by CHK1 inhibitors (9). Noteworthy, a recent study demonstrated that the ATR-Chk1 pathway promoted RRM2 accumulation by CDK2, limiting DNA replication stress and generation of single-stranded DNA (ssDNA) (10). Ribonucleotide reductase is composed of the homodimeric RRM1 and RRM2 subunits that catalyze the conversion of ribonucleotides to deoxyribonucleotides (dNTPs), which are used in the synthesis of DNA during replication and repair (11). We propose that inhibition of Chk1 may enhance sensitization of DNA-damaging agents via suppressing the RR level, exhausting dNTP and enhancing DNA damage.

To explore the contribution of ribonucleotide reductase and DNA damage on chemosensitization by CHK1 inhibitors, we selected a potent inhibitor of CHK1, LY2603618 which has been demonstrated activity both as a monotherapy and in combination with a range of cytotoxic chemotherapeutic agents $(12,13)$. We observed the molecular mechanism of cytotoxic effects of LY2603618 alone and in combination with gemcitabine. 


\section{Materials and methods}

Drugs. LY2603618 and roscovitine were purchased from Selleck Chemicals (Houston, TX, USA). Gemcitabine was purchased from Sigma-Aldrich (St. Louis, MO, USA).

Cell culture. ASPC-1, CFPAC-1, HPAC, BxPC-3 and MiaPaCa-2 cell lines were purchased from the American Type Culture Collection (ATCC; Manassas, VA, USA). The cell lines were cultured in RPMI 1640 medium (Invitrogen, for ASPC-1 and BxPC-3), Dulbecco's modified Eagle's medium (DMEM, Invitrogen, for HPAC and MiaPaCa-2), or Iscove's Modified Dulbecco's medium (IMDM, Invitrogen, for CFPAC-1) containing $10 \%$ fetal bovine serum, $100 \mathrm{u} / \mathrm{ml}$ pencillin, and $100 \mu \mathrm{g} / \mathrm{ml}$ streptomycin in a $37^{\circ} \mathrm{C}$ humidified atmosphere containing $5 \% \mathrm{CO}_{2} / 95 \%$ air. All cell lines were authenticated by the University of Arizona Genetics Core Facility (Tucson, AZ, USA).

Cell viability assay. In vitro cytotoxicities of LY2603618, gemcitabine and roscovitine, alone or in combination, against the pancreatic cancer cell lines were measured using MTT. [3-(4,5-dimethyl-thiazol-2-yl)-2,5-diphenyltetrazolium-bromide, Sigma-Aldrich] assays, as previously described $(14,15) . \mathrm{IC}_{50}$ values were calculated as the drug concentrations necessary to inhibit $50 \%$ proliferation as compared to untreated control cells. The extent and direction of LY2603618 and gemcitabine or roscovitine cytotoxic interactions were determined by standard isobologram analyses, as previously described (15-17).

Cell cycle analysis. Cell cycle distribution was determined by using propidium iodide (PI) staining and flow cytometry analysis with a FACScan flow cytometer (Becton Dickinson, San Jose, CA, USA), as previously described (17). Cell cycle analysis was performed using Multicycle software (Phoenix Flow Systems, Inc., San Diego, CA, USA).

Western blot analysis. Western blotting was performed using polyvinylidene difluoride (PVDF) membranes (Thermo Fisher Inc., Rockford, IL, USA) and iimmunoblotted with mouse antiChk1 (sc8408, 1:500; Santa Cruz Biotechnology, Santa Cruz, CA, USA), and - $\beta$-actin antibodies (A2228/A5441, mouse, 1:2,500; Sigma-Aldrich), or rabbit anti-PARP (9542, 1:1,000), -pCDK1(Y15) (9111, 1:2,000), -CDK2 (2546, 1:2,000), $-\gamma \mathrm{H} 2 \mathrm{AX}(2577,1: 1,000$; Cell Signaling Technology, Danvers, MA, USA), -RRM1 (ab137114, 1:10,000), -RRM2 (ab172476, 1:2,000), -pCHK1 (S345) (ab47318, 1:500), -pCDC25C (S216) (ab32051, 1:1,000), -pCDK2 (Y15) (ab76146, 1:2,000), -CDK1 (ab32094, 1:1,000), and -cleaved-caspase-3 antibodies (ab2302, 1:1,000; Abcam, Cambridge, MA, USA), as previously described (18). Immunoreactive proteins were visualized using the Odyssey Infrared Imaging System (Li-Cor), as described by the manufacturer.

Alkaline comet assay. Pancreatic cancer cells were treated with LY2603618 and gemcitabine, alone or in combination for $8 \mathrm{~h}$ and then subjected to alkaline comet assay, as previously described (19). Slides were stained with SYBR Gold (Life Technologies), and then visualized using Olympus
IX-70 fluorescence microscope (Olympus, Tokyo, Japan). At least 100 comets per gel were scored using CometScore (TriTekCorp, Sumerduck, VA). The comets were analyzed based on the percentage (\%) of DNA in the tail as the measure of primary DNA damage.

Statistical analysis. Data are expressed as the mean \pm standard deviation of three experiments. Differences in the sample means between test groups and control groups were analyzed using the pair-wise two-sample t-test. Statistical analyses were performed with GraphPad Prism 5.0 (GraphPad Software, Inc.). A P-value of $<0.05$ was considered as significant and labeled as ${ }^{*} \mathrm{P}<0.05 ;{ }^{* *} \mathrm{P}<0.01 ;{ }^{* * *} \mathrm{P}<0.001$

\section{Results}

CHK1 inhibition induces growth inhibition and cell death in pancreatic cancer cells. To evaluate the anti-tumor efficacy of LY2603618 in human pancreatic cancer cells, we selected 5 pancreatic cancer cell lines with different p53 phenotype, BxPC-3 (p53 mutation), MiaPaCa-2 (p53 mutation), HPAC (p53 wild-type), CFPAC (p53 mutation) and ASPC-1 (p53 null). The results showed that LY2603618 inhibited cell proliferation in all studied pancreatic cancer cell lines in a dose-dependent manner after $72 \mathrm{~h}$ of treatment (Fig. 1A). The $\mathrm{IC}_{50}$ values of LY2603618 modestly varied from $0.89 \mu \mathrm{M}$ for HPAC cells to $2.75 \mu \mathrm{M}$ for MiaPaCa-2 cells (Fig. 1B), which are less than the maximum clinically achievable concentration of LY2603618 (9 $\mu \mathrm{M})(13)$.

To explore whether LY2603618 causes pancreatic cancer cell death, we treated BxPC-3 (sensitive to LY2603618 with $\mathrm{IC}_{50}$ of $1.00 \mu \mathrm{M}$ ) and $\mathrm{MiaPaCa}-2$ cells (low sensitive to LY2603618 with $\mathrm{IC}_{50}$ of $2.75 \mu \mathrm{M}$ ) with varying concentrations of LY2603618 for $48 \mathrm{~h}$. No more than $25 \%$ cells with DNA fragments (Sub-G1) were observed after LY2603618 treatment by PI staining followed by flow cytometry (Fig. 1C and D), accompanied by an increased PARP cleavage (Fig. 1E and F). It indicates that LY2603618 causes a small amount of pancreatic cancer cell death.

Inhibition of CHK1 causes CDK-dependent RRM1/2 downregulation and DNA damage in pancreatic cancer cells. To confirm CHK1 inhibition by LY2603618, we analyzed CHK1 signaling in LY2603618-treated pancreatic cancer cells by western blot analysis. First, we determined the phosphorylated and total protein levels of CHK1 after $48 \mathrm{~h}$ of treatment with LY2603618 in clinically achievable concentrations. LY2603618 decreased the total CHK1 level but increased the pCHK1S345 level in BxPC-3 or MiaPaCa-2 cells (Fig. 2A). Since Ser345 phosphoylation is predominantly catalyzed by ATR in response to DNA damage (20), our results suggest that LY2603618 treatment may cause DNA damage-mediated phosphorylation of CHK1 at Ser345. Generally, CDC25C phosphorylation by $\mathrm{CHK} 1$ may predit $\mathrm{CHK} 1$ activity. Next, we observed CHK1 downstream signaling effectors, including pCDC25C, CDK1, pCDK1, CDK2, and pCDK2. The results showed that LY2603618 treatment reduced the phosphorylated protein level of CDC25C, CDK1, and CDK2 without altering the total protein levels of CDK1 and CDK2 in BxPC-3 or MiaPaCa-2 cells (Fig. 2A), indicating that LY2603618 inhibites CHK1 activity and activated CDK1/2. 

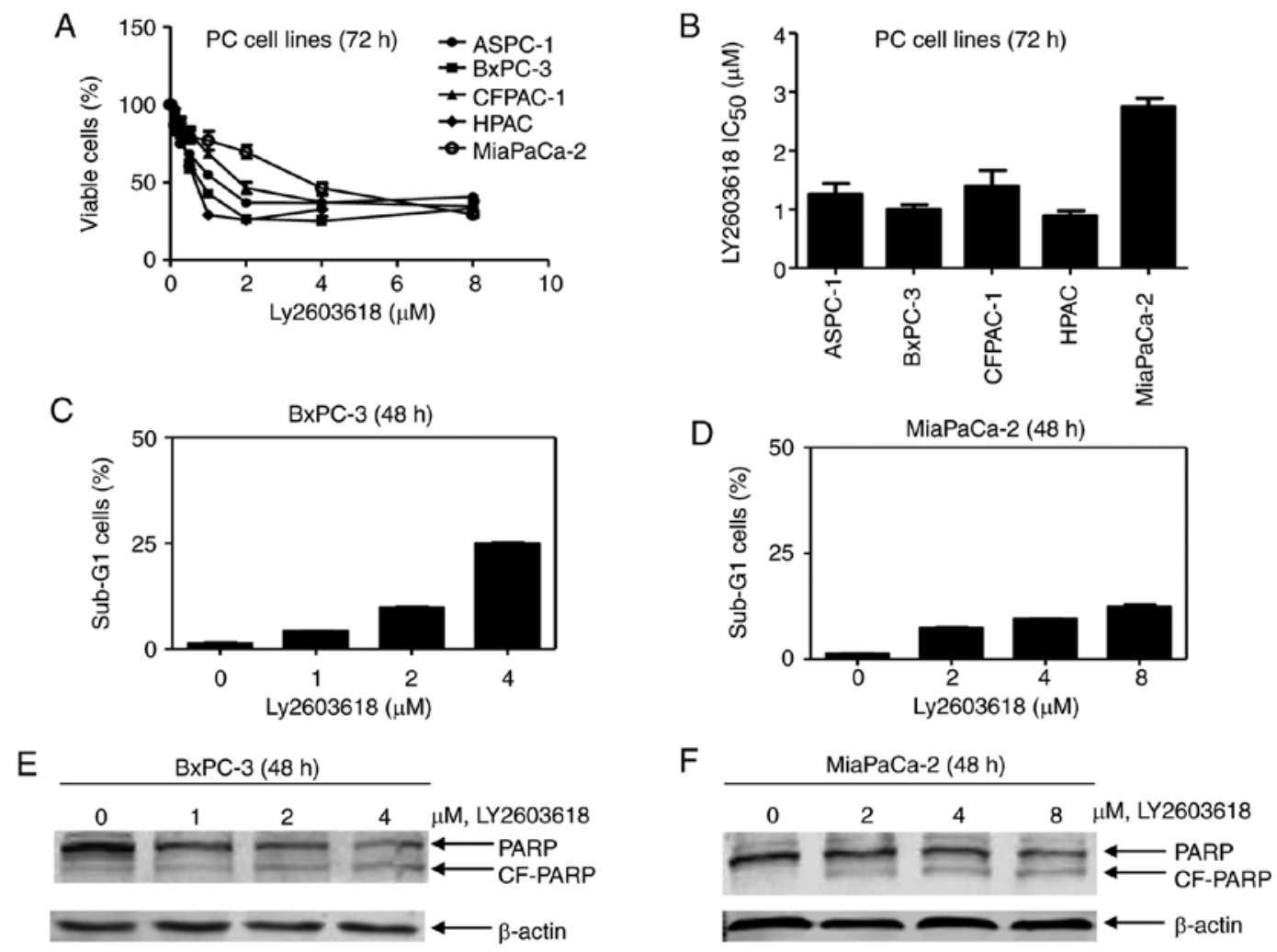

Figure 1. LY2603618 induces growth inhibition and cell death in pancreatic cancer cells. (A and B) Pancreatic cancer cell lines were treated with variable concentrations of LY2603618 for $72 \mathrm{~h}$ and viable cells were determined using MTT reagent (A). $\mathrm{IC}_{50}$ values were calculated as drug concentration necessary to inhibit 50\% proliferation as compared to untreated control cells (B). Data are graphed as mean values \pm SEM from three independent experiments. BxPC-3 (C) and MiaPaCa-2 (D) cells were treated with varying concentrations of LY2603618 for $48 \mathrm{~h}$ and then subjected to PI staining and flow cytometry analyses. Dead cells are expressed as the percentage of $\mathrm{PI}^{+}$cells with sub-G1 DNA content. Data are presented as the mean of triplicate experiments \pm SEM. BxPC-3 (E) and MiaPaCa-2 (F) cells were treated with LY2603618 for $48 \mathrm{~h}$. Whole cell lysates were subjected to western blotting and probed with anti-PARP or - $\beta$-actin antibodies. Experiments were performed at least 3 independent times, and representative western blots are shown.

Since inhibition of CHK1 may suppress RRM2 expression, leading to DNA replication stress and DNA damage, we next observed effect of LY2603618 on RRM1 and RRM2 levels in BxPC-3 and MiaPaCa-2 cells. As shown in Fig. 2A, LY2603618 treatment decreased the protein levels of RRM1 and RRM2, accompanied by a dose-dependent increase of phosphorylated $\mathrm{H} 2 \mathrm{AX}(\gamma \mathrm{H} 2 \mathrm{AX}$, an established biomarker for DNA double-strand breaks) in both cell lines. Time course experiments demonstrated that decreases of CHK1, pCDC25C, pCDK1, pCDK2 and RRM1/2 protein levels and increases of pCHK1 and $\gamma \mathrm{H} 2 \mathrm{AX}$ were simultaneously detected as early as $4 \mathrm{~h}$ in BxPC-3 cells and as early as $8 \mathrm{~h}$ in MiaPaCa-2 cells (Fig. 2B). This finding indicates that LY2603618-induced CHK1 inhibition, CDK activation, RRM1/2 downregulation and DNA damage simultaneously occur at an earlier time in pancreatic cancer cells.

To determine whether RRM1/2 downregulation and DNA damage are dependent on CDK activation in response to LY2603618, we selected a CDK1/2/5 inhibitor, roscovitine. Noteworthy, roscovitine almost completely restored the levels of RRM1/2 and $\gamma \mathrm{H} 2 \mathrm{AX}$ in LY2603618-treated cells (Fig. 2C and D), suggesting that CHK1 inhibition causes CDK-dependent RRM1/2 downregulation and DNA damage in pancreatic cancer cells. Furthermore, we observed the effect of roscovitine on LY2603618-induced cytotoxicity in BxPC-3 and $\mathrm{MiaPaCa}-2$ cells. As expected, roscovitine significantly decreased the amount of Sub-G1 cells in the presence of
LY2603618 (Fig. 2E and F). Moreover, when administered simultaneously, roscovitine significantly attenuated LY2603618 sensitivities reflected by increased $\mathrm{IC}_{50}$ values in BxPC-3 and MiaPaCa-2 cells (Fig. 2G and H). The combined effects of LY2603618 with roscovitine on cell proliferation were clearly antagonistic, reflected by all points falling above the line using standard isobologram analysis. Taken together, our data indicate that CHK1 inhibition by LY2603618 causes CDK-dependent RRM1/2 downregulation, DNA damage, and cytotoxicity in pancreatic cancer cells.

CHKI inhibition synergizes with gemcitabine treatment to induce growth inhibition and apoptosis in pancreatic cancer cells. Since interference with DNA damage checkpoints has been demonstrated preclinically to be a highly effective means of increasing the cytotoxicity of DNA-damaging drugs, we then investigated the combination of LY2603618 with gemcitabine. When simultaneously administered with LY2603618, gemcitabine significantly enhanced LY2603618 sensitivity, reflected by the decreased $\mathrm{IC}_{50}$ values in 5 pancreatic cancer cell lines (Fig. 3A-E). The combined effects of LY2603618 with gemcitabine on cell proliferation were clearly synergistic, reflected by all the points falling below the line by standard isobologram analysis (Fig. 3A-E).

To further address the synergism of LY2603618 and gemcitabine, we treated the BxPC- 3 or MiaPaCa- 2 cells with both drugs alone or in combination and looked at their effects 
A
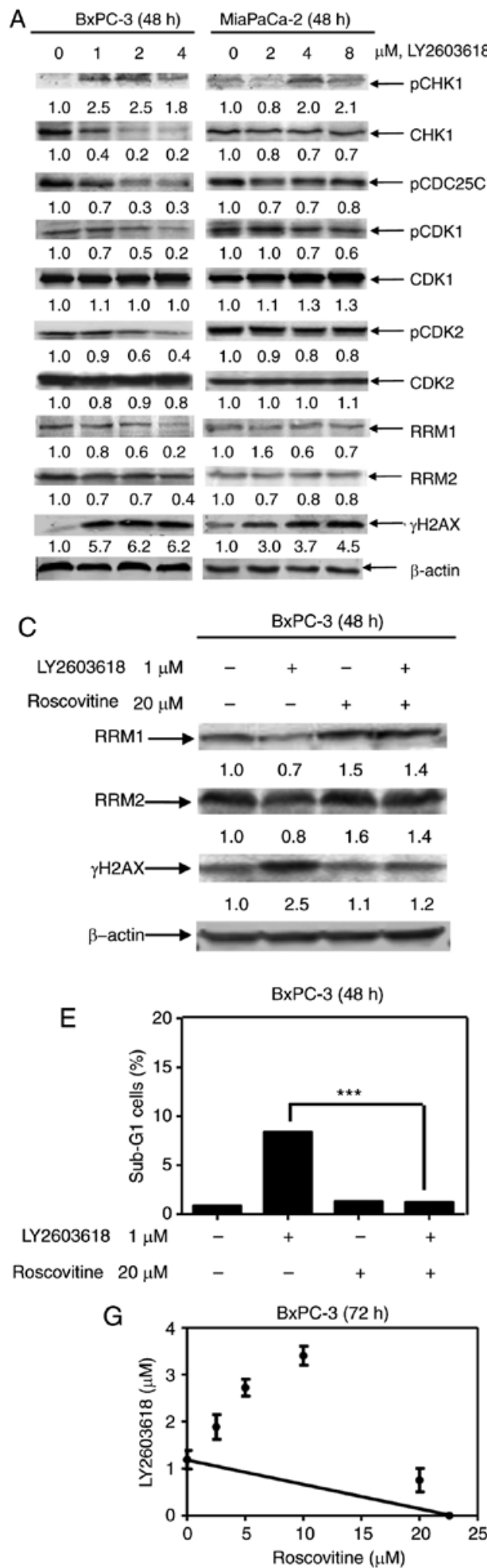

B

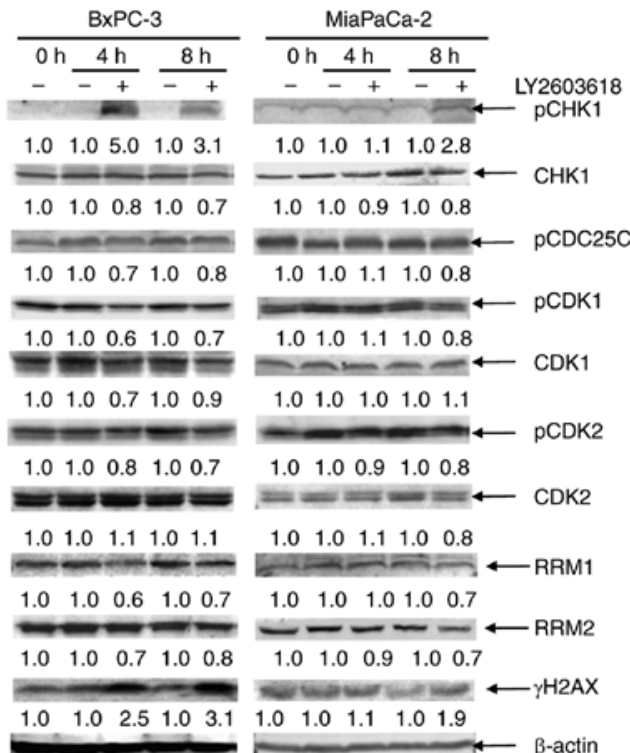

D

MiaPaCa-2 (48 h)
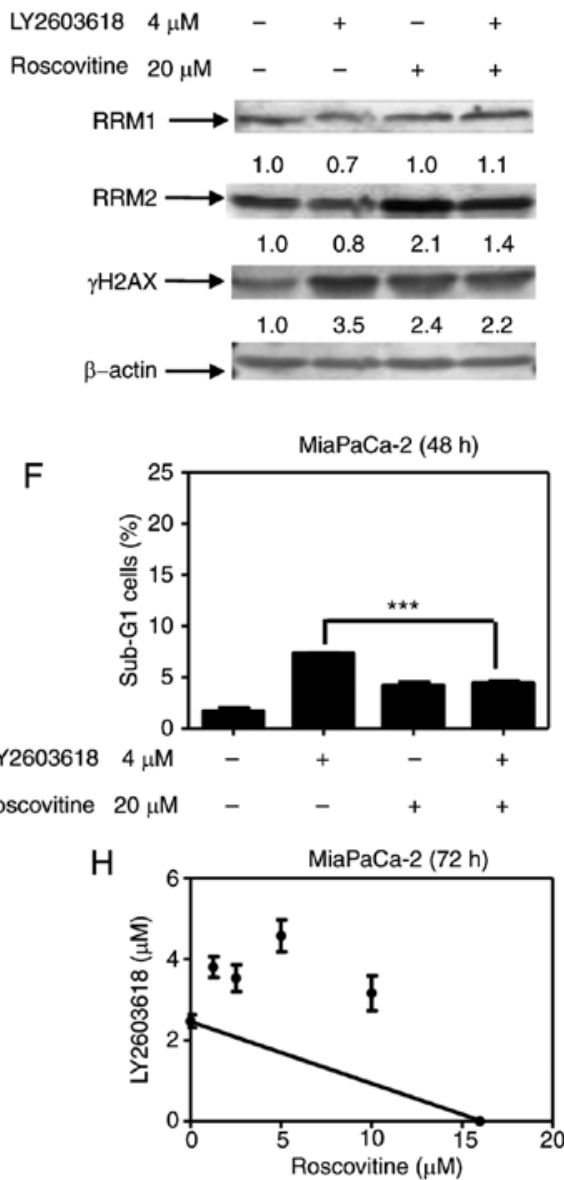

Figure 2. LY2603618 causes CDK-dependent RRM1/2 downregulation and DNA damage enhancement in pancreatic cancer cells. (A) BxPC-3 and MiaPaCa-2 cells were treated with varying concentrations of LY2603618 for $48 \mathrm{~h}$. Whole cell lysates were subjected to western blotting and probed with anti-pCHK1, -CHK1, -pCDC25C, -pCDK1, -CDK1, -pCDK2, -CDK2, -RRM1, -RRM2, - $\gamma$ H2AX or - $\beta$-actin antibodies. (B) After 4 and 8 h of LY2603618 treatment of BxPC-3 $(1 \mu \mathrm{M})$ and MiaPaCa-2 $(4 \mu \mathrm{M})$, cells were harvested and lysed. Protein extracts were subjected to western blotting and probed with anti-pCHK1, -CHK1, -pCDC25C, -pCDK1, -CDK1, -pCDK2, -CDK2, -RRM1, -RRM2, - $\gamma \mathrm{H} 2 \mathrm{AX}$ or - $\beta$-actin antibodies. All experiments were performed at least 3 independent times, and representative western blots are shown. (C-F) BxPC-3 or MiaPaCa-2 cells were treated with 1 or $4 \mu \mathrm{M} \mathrm{LY} 2603618$ in the absence or presence of $20 \mu \mathrm{M}$ roscovitine for $48 \mathrm{~h}$, respectively. RRM1, RRM2, $\gamma \mathrm{H} 2 \mathrm{AX}$ or $-\beta$-actin protein levels in BxPC-3 (C) and MiaPaCa-2 (D) cells were shown by western blot analysis. All experiments were performed at least 3 independent times, and representative western blots are shown. The percentage of $\mathrm{PI}^{+}$cells with sub-G1 DNA content in BxPC-3 (E) and MiaPaCa-2 (F) cells was measured by PI staining and flow cytometry analyses. ${ }^{* * *} \mathrm{P}<0.001$. BxPC-3 (G) and MiaPaCa-2 (H) cells were treated with LY2603618 and roscovitine, alone or in combination, for $72 \mathrm{~h}$ and then viable cells were determined using MTT reagent. Standard isobologram was used to analyze the antagonistic cytotoxic effect of LY2603618 and roscovitine. The $\mathrm{IC}_{50}$ values of each drug are plotted on the axes; the solid line represents the additive effect, while the points represent the concentrations of each drug resulting in 50\% inhibition of proliferation. Points falling below the line indicate synergism whereas those above the line indicate antagonism. 

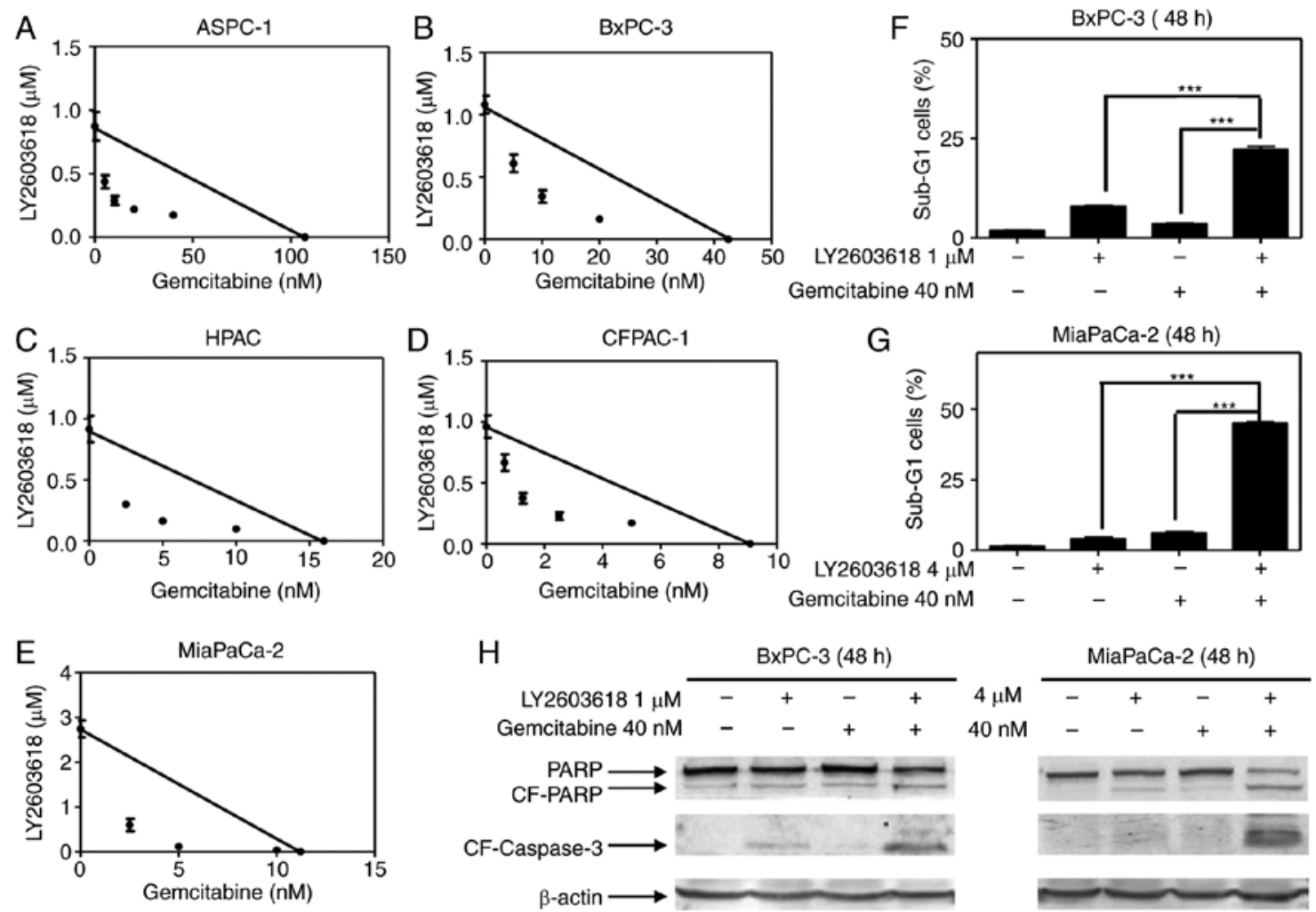

Figure 3. LY2603618 synergizes with gemcitabine treatment to induce growth inhibition and apoptosis in pancreatic cancer cells. ASPC-1 (A), BxPC-3 (B), HPAC (C), CFPAC-1 (D) and MiaPaCa-2 (E) cells were treated with LY2603618 and gemcitabine, alone or in combination, for $72 \mathrm{~h}$ and then viable cells were determined using MTT reagent. Standard isobologram was used to analyze the synergistic cytotoxicity of LY2603618 and gemcitabine. BxPC-3 (F) and MiaPaCa-2 (G) cells were treated with vehicle control, gemcitabine (40 nM), LY2603618 (1 $\mu \mathrm{M}$ for BxPC-3 or $4 \mu \mathrm{M}$ for MiaPaCa-2) or gemcitabine plus LY2603618 for $48 \mathrm{~h}$. Then cells were subjected to PI staining and flow cytometry analyses. Dead cells are expressed as the percentage of PI cells with sub-G1 DNA content. Data are presented as the mean of triplicate experiments \pm SEM. ${ }^{* * * *} \mathrm{P}<0.001$. (H) Cells were treated as shown in $(\mathrm{F}$ and G). Then whole cell lysates were subjected to western blotting and probed with anti-PARP, -cleaved-caspase- 3 or $-\beta$-actin antibodies. Experiments were performed at least 3 independent times, and representative western blots are shown.

on cell apoptosis. The results showed that LY2603618 and gemcitabine cooperatively induced apoptosis, as indicated by the high percentage of cells with sub-G1 DNA content and the increased cleavage of PARP and caspase-3 (Fig. 3F-H).

Gemcitabine sensitization by CHK1 inhibition is associated with CDK-dependent RRMI/2 downregulation and DNA damage enhancement. To explore the molecular mechanism of gemcitabine sensitization by LY2603618, cell cycle progression was analyzed by flow cytometry. Gemcitabine treatment led to $\mathrm{S}$ and $\mathrm{G} 2 / \mathrm{M}$ arrest, which was abrogated to some extent by the addition of LY2603618 in both pancreatic cancer cells (Fig. 4A and B). It is in accordance with the western blotting results that LY2603618 inhibited gemcitabine-induced pCDC25C, CDK1/2 and pCDK1/2 protein levels (Fig. 4C). Collectively, it suggests that inhibition of Chk1 abrogates gemcitabine-activated $\mathrm{S}$ and $\mathrm{G} 2 / \mathrm{M}$ checkpoints.

We next looked at DNA damage in the combined treatment of pancreatic cancer cells. As expected, gemcitabine treatment increased $\gamma \mathrm{H} 2 \mathrm{AX}$ level in both BxPC-3 and MiaPaCa-2 cell lines, which was further increased by the addition of LY2603618, indicating that DNA damage was enhanced by the combined treatment (Fig. 4D). Meanwhile, gemcitabine treatment caused modest increase of both RRM1 and RRM2, which were further decreased by LY2603618 (Fig. 4D). Noteworthy, roscovitine treatment almost completely restored the levels of RRM1/2 and $\gamma \mathrm{H} 2 \mathrm{AX}$ in the combined treatment of pancreatic cancer cells (Fig. 5A). More importantly, after the combined treatment of gemcitabine and LY2603618, the amount of Sub-G1 cells was significantly decreased by roscovitine, accompanied with the downregulation of cleaved PARP (Fig. 5A-C). Taken together, it suggests that gemcitabine sensitization by $\mathrm{CHK} 1$ inhibition is associated with CDK-dependent RRM1/2 downregulation and DNA damage enhancement.

To determine whether DNA damage occurs prior to induction of apoptosis in response to LY2603618 and gemcitabine, the cells were treated for a shorter time, $8 \mathrm{~h}$, and then DNA damage was determined by the alkaline comet assay. As demonstrated in Fig. 6A-D, LY2603618 significantly increased the percentage of DNA in the tail and the $\gamma \mathrm{H} 2 \mathrm{AX}$ level in gemcitabine-treated pancreatic cancer cells. However, cleaved PARP was not detected after the combination treatment, providing evidence that gemcitabine combined with LY2603618 caused increased DNA damage, prior to induction of apoptosis.

\section{Discussion}

In the past several years, various specific small molecule CHK1 inhibitors have been developed. Understanding the mechanisms of action of these inhibitors may help to guide their application in clinical settings. In the study, we 


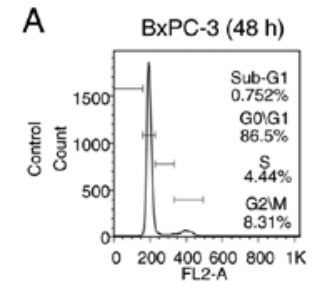

B MiaPaCa-2 $(48 \mathrm{~h})$
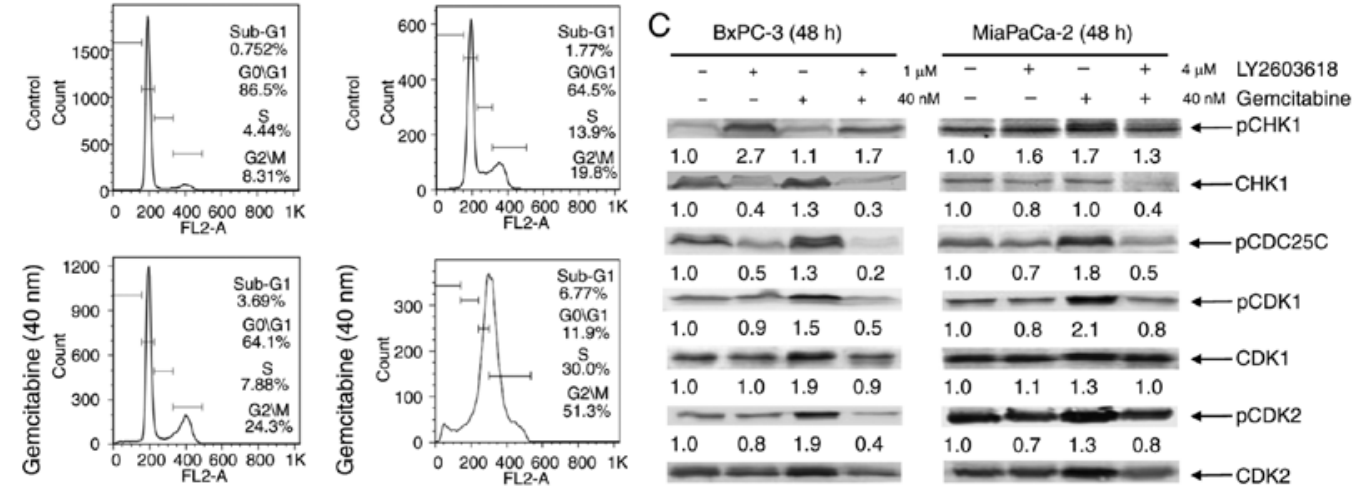

$\begin{array}{llllllll}1.0 & 0.5 & 1.3 & 0.2 & 1.0 & 0.7 & 1.8 & 0.5\end{array} \mathrm{PCDC} 25 \mathrm{C}$

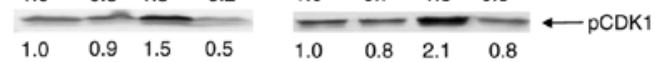

$\longrightarrow$

$\begin{array}{llll}1.0 & 1.0 & 1.9 & 0.9\end{array}$

$\frac{1.0}{1.0} \frac{0.8}{1.3} \frac{2.1}{1.0} \leftarrow \mathrm{CDK} 1$

$\begin{array}{lllll}1.0 & 0.8 & 1.9 & 0.4\end{array}$

\begin{tabular}{llll}
1.0 & 0.8 & 1.9 & 0.4 \\
\hline & $=$ & 0.9
\end{tabular}

$\longleftarrow$ ־CDK2

\begin{tabular}{llll}
1.0 & 0.7 & 1.3 & 0.8 \\
\hline
\end{tabular}
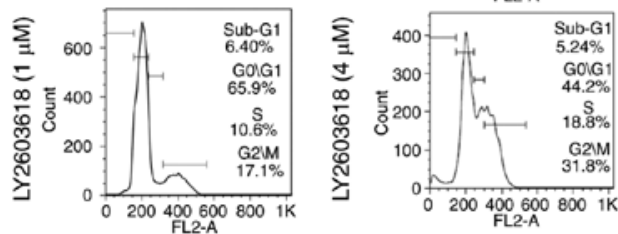

\begin{tabular}{llll}
1.0 & 0.9 & 1.8 & 0.6 \\
\hline
\end{tabular}

$\begin{array}{llll}1.0 & 1.0 & 1.8 & 0.9\end{array}$

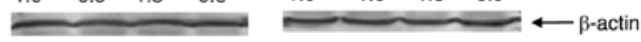

D $\quad \operatorname{BxPC}-3(48 \mathrm{~h})$
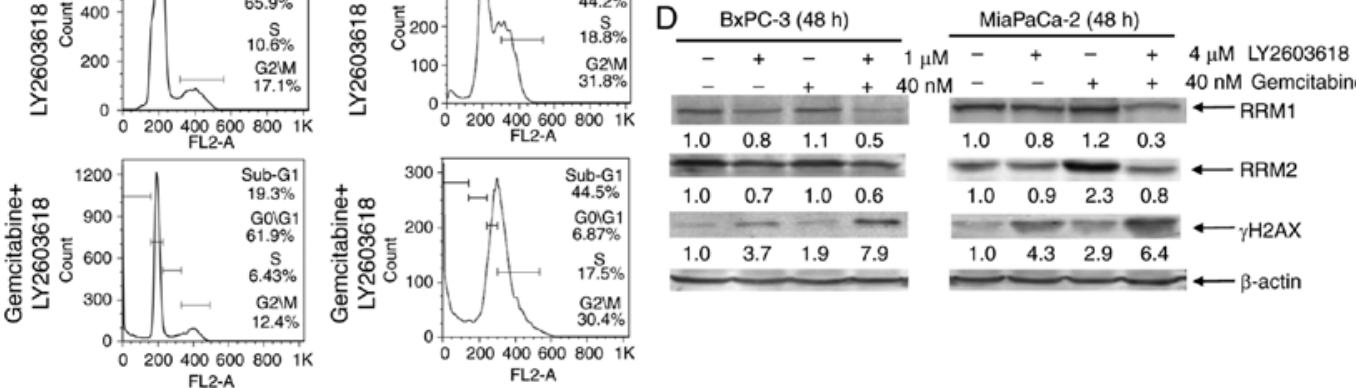

Figure 4. LY2603618 abrogates gemcitabine-activated S and G2/M checkpoint and inhibits gemcitabine-induced RRM1/2 level in pancreatic cancer cells. BxPC-3 (A) and MiaPaCa-2 (B) cells were treated with vehicle control, gemcitabine (40 nM), LY2603618 (1 $\mu \mathrm{M}$ for BxPC-3 or $4 \mu \mathrm{M}$ for MiaPaCa-2) or gemcitabine plus LY2603618 for $48 \mathrm{~h}$. Cell cycle distribution was detected by PI staining and flow cytometry analyses. (C and D) Cells were treated as shown in (A and B). pCHK1, CHK1, pCDC25C, pCDK1, CDK1, pCDK2, CDK2or $\beta$-actin protein levels in BxPC-3 and MiaPaCa-2 cells were detected by western blot analysis (C). RRM1, RRM2, $\gamma \mathrm{H} 2 \mathrm{AX}$ or $\beta$-actin protein levels in BxPC-3 and MiaPaCa-2 cells were detected by western blot analysis (D). Experiments were performed at least 3 independent times, and representative western blots are shown.

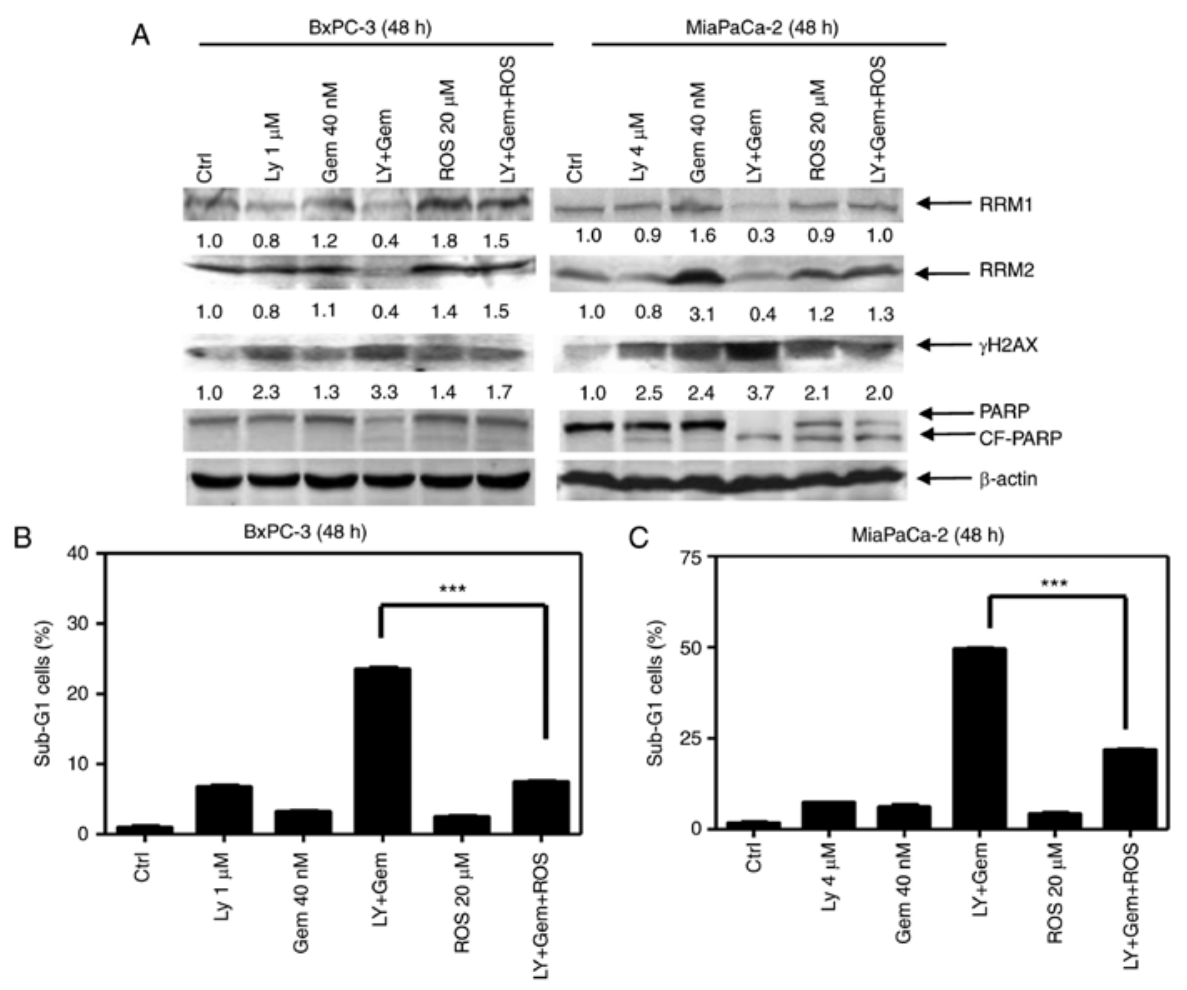

Figure 5. Roscovitine treatment reverses the cytotoxic effects of gemcitabine combined with LY2603618. (A-C) BxPC-3 and MiaPaCa-2 cells were treated with vehicle control (Ctrl), gemcitabine (Gem, $40 \mathrm{nM}$ ), LY2603618 (LY, $1 \mu \mathrm{M}$ for BxPC-3 or $4 \mu \mathrm{M}$ for MiaPaCa-2), gemcitabine plus LY2603618 (Gem+LY), roscovitine (ROS, $20 \mu \mathrm{M}$ ), or gemcitabine plus LY2603618 plus roscovitine (Gem+LY+ROS) for $48 \mathrm{~h}$. RRM1, RRM2, $\gamma \mathrm{H} 2 \mathrm{AX}$, PARP or $\beta$-actin protein levels in BxPC-3 and MiaPaCa-2 cells were detected by western blot analysis (A). Experiments were performed at least 3 independent times, and representative western blots are shown. The percentage of Sub-G1 cells in BxPC-3 (B) and MiaPaCa-2 cells (C) were calculated by PI staining and flow cytometry analyses. Data are presented as the mean of triplicate experiments \pm SEM. ${ }^{* * *} \mathrm{P}<0.001$. 


\section{A}

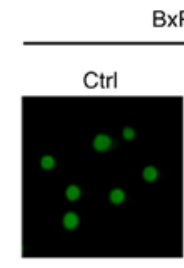

$\mathrm{BxPC}-3(8 \mathrm{~h})$

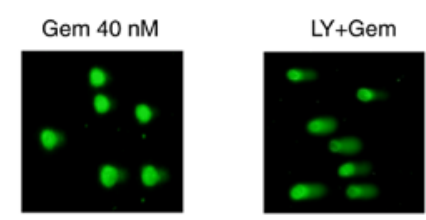

C
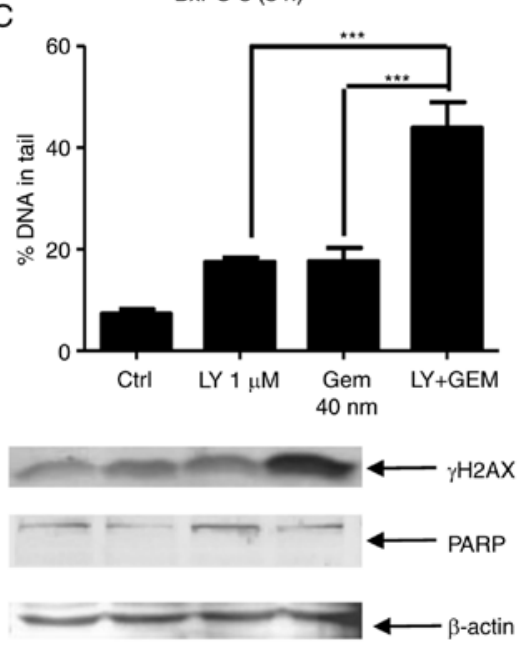

$\mathrm{B}$

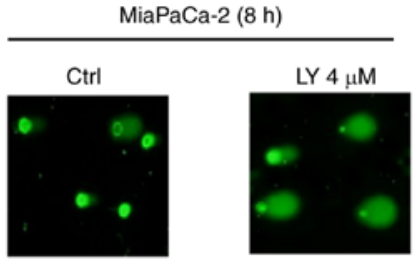

Gem $40 \mathrm{nM}$
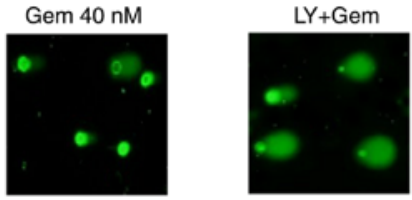

D
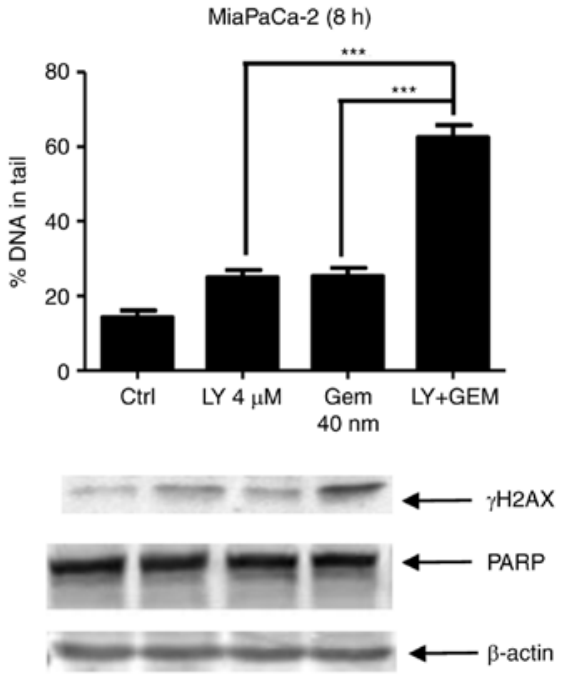

Figure 6. Gemcitabine in combination with LY2603618 causes increased DNA damage, prior to induction of apoptosis. BxPC-3 (A) and MiaPaCa-2 (B) cells were treated with vehicle control (Ctrl), gemcitabine (Gem, $40 \mathrm{nM}$ ), LY2603618 (LY, $1 \mu \mathrm{M}$ for BxPC-3 or $4 \mu \mathrm{M}$ for MiaPaCa-2), gemcitabine plus LY2603618 $(\mathrm{Gem}+\mathrm{LY})$ for $8 \mathrm{~h}$ and then subjected to alkaline comet assay analysis. Representative images are shown. (C and D) Comet assay results are graphed as median percent DNA in the tail from 3 replicate gels \pm SEM. ${ }^{* * *} \mathrm{P}<0.001$. Meanwhile, $\gamma \mathrm{H} 2 \mathrm{AX}$, PARP or $\beta$-actin protein levels in BxPC-3 and MiaPaCa-2 cells were detected by western blot analysis. Experiments were performed at least 3 independent times, and representative western blots are shown.

demonstrate that inhibition of CHK1 by LY2603618 potentiates anti-pancreatic cancer activity of gemcitabine through promoting CDK-dependent ribonucleotide reductase downregulation and DNA damage.

First, our results showed that LY2603618 treatment of pancreatic cancer cells caused growth inhibition and a small amount of cell death, which were alleviated by a CDK1/2/5 inhibitor, roscovitine. This indicates that the cytotoxic effect of LY2603618 is dependent on CDK activation. Consistently, CDK1/2 activation was observed in LY2603618-treated pancreatic cancer cells, as evidenced by the reduced pCDC25C, pCDK1, and pCDK2 levels. Further, we found that LY2603618 treatment reduced the levels of RRM1 and RRM2, resulting in DNA damage, which was also completely reversed by roscovitine. It suggests that activation of $\mathrm{CDK}$ by $\mathrm{CHK} 1$ inhibition may reduce RRM1/2 accumulation, leading to DNA damage, consistent with a previous report (10). Collectively, we infer that Chk1 inhibition causes cytotoxicity in pancreatic cancer cells by promoting CDK-dependent RRM1/2 downregulation and DNA damage.

Of note, we found that LY2603618 significantly increased the phosphorylated CHK1S345 level, consistent with a previous study (20). It is reported that phosphorylation of Chk1 at S345 is predominantly catalyzed by ATR in response to DNA damage $(21,22)$, indicating that enhanced pCHK1S345 level may be attributed to DNA damage caused by LY2603618. As expected, we observed the enhanced $\gamma \mathrm{H} 2 \mathrm{AX}$ levels in dose-dependant manner after 48 h of LY2603618 treatment. Furthermore, a time course experiment showed that the protein levels of $\gamma \mathrm{H} 2 \mathrm{AX}$ and pCHK1S345 were simultaneously increased by LY2603618 treatment ( $4 \mathrm{~h}$ for BxPC-3 cells and $8 \mathrm{~h}$ for MiaPaCa-2 cells). Noteworthy, in contrast to the increased pCHK1S345 protein level, the decreased total CHK1 levels were also detected in LY2603618-treated cells. This may be explained by a previous report demonstrating that ATR-mediated phosphorylation of CHK1 at S345 induced the polyubiquitination and degradation of CHK1 in human cells (23). Taken together, these data support our hypothesis that LY2603618 causes CDK-dependent DNA damage, which further activates the ATR-CHK1 pathway, resulting in CHK1 phosphorylation at S345 and subsequently CHK1 degradation in pancreatic cancer cells.

As a key activator of the S- or G2/M- phase DNA damage response, CHK1 may be involved in gemcitabine resistance in cancer therapy. Thus interference with DNA damage checkpoints by CHK1 inhibition has been demonstrated to be an effective means of increasing the cytotoxicity of gemcitabine. As expected, we observed synergistic anti-pancreatic cancer 
activities of LY2603618 and gemcitabine in 5 pancreatic cancer cell lines with different p53 phenotype, consistent with several preclinical studies (24-26), suggesting that p53 status does not play a major role in sensitization of gemcitabine by LY2603618. Moreover, the combination induced pronounced levels of apoptosis as indicated by an increase in the fraction of sub-G1 cells or in the levels of cleaved PARP and caspase-3. Mechanistic investigations showed that $\mathrm{CHK} 1$ inhibition by LY2603618 partially abrogated S and G2/M phase checkpoints and significantly enhanced DNA damage in gemcitabine-treated pancreatic cancer cells, which is consistent with an in vivo study (26). This suggests that the abrogation of $\mathrm{S}$ or $\mathrm{G} 2 / \mathrm{M}$ checkpoint contributes to sensitization of gemcitabine by LY2603618. In addition, it is reported that one of molecular mechanisms of gemcitabine resistance includes upregulation of gemcitabine targets, RRM1 and RRM2, which play an essential role in the maintenance of the dNTPs level $(27,28)$. RRM1 has been identified as the major determinant of gemcitabine efficacy in patients treated with gemcitabine. Interestingly, our results showed that RRM1 and RRM2 protein levels were much lower after the combined treatment than after LY2603618 or gemcitabine treatment alone. It suggests that downregulation of RRM1/2 by LY2603618 potentiates the effect of gemcitabine by decreasing the competition between gemcitabine and deoxycytidine, and then increasing DNA damage. To test whether CDK activation contributes to RRM1/2 downregulation and DNA damage by the combined treatment, we added CDK inhibitor, roscovitine. Surprisingly, RRM1/2 and $\gamma \mathrm{H} 2 \mathrm{AX}$ levels were almost completely restored after $48 \mathrm{~h}$ of roscovitine treatment. Furthermore, roscovitine significantly decreased the amount of Sub-G1 cells in combined treatment of pancreatic cancer cells. These data reveal that activation of CDK at least partly contributes to synergistic cytotoxicity of gemcitabine and LY2603618 by decreasing RRM1/2 protein level and enhancing DNA damage.

In conclusion, CHK1 inhibition by LY2603618 treatment caused a CDK-dependent cell death via downregulating RRM1/2 levels and enhancing DNA damage. Combined use of gemcitabine and LY2603618 synergistically reduced pancreatic cancer cell viability relative to either single treatment, which was also associated with CDK-dependent RRM1/2 downregulation and DNA damage enhancement. These findings suggest that CDK activation plays an important role in cytotoxicities of $\mathrm{CHK} 1$ inhibitor alone or in combination with gemcitabine in pancreatic cancer cells.

\section{Acknowledgements}

This project was supported by the Science and Technology Development Program of Jilin Province (no. 20150101185JC).

\section{References}

1. Jemal A, Siegel R, Ward E, Hao Y, Xu J, Murray T and Thun MJ: Cancer statistics, 2008. CA Cancer J Clin 58: 71-96, 2008.

2. Burris HA III, Moore MJ, Andersen J, Green MR, Rothenberg ML, Modiano MR, Cripps MC, Portenoy RK, Storniolo AM, Tarassoff $\mathrm{P}$, et al: Improvements in survival and clinical benefit with gemcitabine as first-line therapy for patients with advanced pancreas cancer: A randomized trial. J Clin Oncol 15: 2403-2413, 1997.
3. Huang P, Chubb S, Hertel LW, Grindey GB and Plunkett W: Action of 2',2'-difluorodeoxycytidine on DNA synthesis. Cancer Res 51: 6110-6117, 1991.

4. Kullmann F, Hollerbach S, Dollinger MM, Harder J, Fuchs M, Messmann H, Trojan J, Gäbele E, Hinke A, Hollerbach C and Endlicher E: Cetuximab plus gemcitabine/oxaliplatin (GEMOXCET) in first-line metastatic pancreatic cancer: A multicentre phase II study. Br J Cancer 100: 1032-1036, 2009

5. Li J and Saif MW: Advancements in the management of pancreatic cancer. JOP 10: 109-117, 2009.

6. Dai Y and Grant S: New insights into checkpoint kinase 1 in the DNA damage response signaling network. Clin Cancer Res 16: 376-383, 2010.

7. Löffler H, Rebacz B, Ho AD, Lukas J, Bartek J and Kramer A: Chk1-dependent regulation of Cdc25B functions to coordinate mitotic events. Cell Cycle 5: 2543-2547, 2006.

8. Scarpa A, Capelli P, Mukai K, Zamboni G, Oda T, Iacono $\mathrm{C}$ and Hirohashi S: Pancreatic adenocarcinomas frequently show p53 gene mutations. Am J Pathol 142: 1534-1543, 1993.

9. Morgan MA, Parsels LA, Zhao L, Parsels JD, Davis MA, Hassan MC, Arumugarajah S, Hylander-Gans L, Morosini D, Simeone DM, et al: Mechanism of radiosensitization by the Chk1/2 inhibitor AZD7762 involves abrogation of the G2 checkpoint and inhibition of homologous recombinational DNA repair. Cancer Res 70: 4972-4981, 2010.

10. Buisson R, Boisvert JL, Benes CH and Zou L: Distinct but concerted roles of ATR, DNA-PK, and Chk1 in countering replication stress durings phase. Mol Cell 59: 1011-1024, 2015.

11. Nordlund P and Reichard P: Ribonucleotide reductases. Annu Rev Biochem 75: 681-706, 2006.

12. King C, Diaz H, Barnard D, Barda D, Clawson D, Blosser W, Cox K, Guo S and Marshall M: Characterization and preclinical development of LY2603618: A selective and potent Chk1 inhibitor. Invest New Drugs 32: 213-226, 2014.

13. Calvo E, Chen VJ, Marshall M, Ohnmacht U, Hynes SM, Kumm E, Diaz HB, Barnard D, Merzoug FF, Huber L, et al: Preclinical analyses and phase I evaluation of LY2603618 administered in combination with pemetrexed and cisplatin in patients with advanced cancer. Invest New Drugs 32: 955-968, 2014.

14. Taub JW, Huang X, Matherly LH, Stout ML, Buck SA, Massey GV, Becton DL, Chang MN, Weinstein HJ and Ravindranath Y: Expression of chromosome 21-localized genes in acute myeloid leukemia: Differences between Down syndrome and non-Down syndrome blast cells and relationship to in vitro sensitivity to cytosine arabinoside and daunorubicin. Blood 94: 1393-1400, 1999.

15. Xie C, Edwards H, Xu X, Zhou H, Buck SA, Stout ML, Yu Q, Rubnitz JE, Matherly LH, Taub JW and Ge Y: Mechanisms of synergistic antileukemic interactions between valproic acid and cytarabine in pediatric acute myeloid leukemia. Clin Cancer Res 16: 5499-5510, 2010.

16. Chou TC: Theoretical basis, experimental design, and computerized simulation of synergism and antagonism in drug combination studies. Pharmacol Rev 58: 621-681, 2006.

17. Wang G, He J, Zhao J, Yun W, Xie C, Taub JW, Azmi A, Mohammad RM, Dong Y, Kong W, et al: Class I and class II histone deacetylases are potential therapeutic targets for treating pancreatic cancer. PLoS One 7: e52095, 2012.

18. Edwards H, Xie C, LaFiura KM, Dombkowski AA, Buck SA, Boerner JL, Taub JW, Matherly LH and Ge Y: RUNX1 regulates phosphoinositide 3-kinase/AKT pathway: Role in chemotherapy sensitivity in acute megakaryocytic leukemia. Blood 114: 2744-2752, 2009.

19. Xie C, Drenberg C, Edwards H, Caldwell JT, Chen W, Inaba H, Xu X, Buck SA, Taub JW, Baker SD and Ge Y: Panobinostat enhances cytarabine and daunorubicin sensitivities in AML cells through suppressing the expression of BRCA1, CHK1, and Rad51. PLoS One 8: e79106, 2013.

20. Wang FZ, Fei HR, Cui YJ, Sun YK, Li ZM, Wang XY, Yang XY, Zhang JG and Sun BL: The checkpoint 1 kinase inhibitor LY2603618 induces cell cycle arrest, DNA damage response and autophagy in cancer cells. Apoptosis 19: 1389-1398, 2014.

21. Kasahara K, Goto H, Enomoto M, Tomono Y, Kiyono T and Inagaki M: 14-3-3gamma mediates Cdc25A proteolysis to block premature mitotic entry after DNA damage. EMBO J 29: 2802-2812, 2010.

22. Clarke CA and Clarke PR: DNA-dependent phosphorylation of Chk1 and Claspin in a human cell-free system. Biochem J 388: 705-712, 2005. 
23. Zhang YW, Otterness DM, Chiang GG, Xie W, Liu YC, Mercurio F and Abraham RT: Genotoxic stress targets human Chk1 for degradation by the ubiquitin-proteasome pathway. Mol Cell 19: 607-618, 2005.

24. Parsels LA, Morgan MA, Tanska DM, Parsels JD, Palmer BD, Booth RJ, Denny WA, Canman CE, Kraker AJ, Lawrence TS and Maybaum J: Gemcitabine sensitization by checkpoint kinase 1 inhibition correlates with inhibition of a Rad51 DNA damage response in pancreatic cancer cells. Mol Cancer Ther 8: 45-54, 2009.

25. Matthews DJ, Yakes FM, Chen J, Tadano M, Bornheim L, Clary DO, Tai A, Wagner JM, Miller N, Kim YD, et al: Pharmacological abrogation of S-phase checkpoint enhances the anti-tumor activity of gemcitabine in vivo. Cell Cycle 6: 104-110, 2007.
26. Barnard D, Diaz HB, Burke T, Donoho G, Beckmann R, Jones B, Barda D, King C and Marshall M: LY2603618, a selective CHK1 inhibitor, enhances the anti-tumor effect of gemcitabine in xenograft tumor models. Invest New Drugs 34: 49-60, 2016.

27. Davidson JD, Ma L, Flagella M, Geeganage S, Gelbert LM and Slapak CA: An increase in the expression of ribonucleotide reductase large subunit 1 is associated with gemcitabine resistance in non-small cell lung cancer cell lines. Cancer Res 64: 3761-3766, 2004.

28. Bergman AM, Eijk PP, Ruiz van Haperen VW, Smid K, Veerman G, Hubeek I, van den Ijssel P, Ylstra B and Peters GJ: In vivo induction of resistance to gemcitabine results in increased expression of ribonucleotide reductase subunit M1 as the major determinant. Cancer Res 65: 9510-9516, 2005. 